\title{
Endomyocardial fibrosis
}

\section{Chukwuemeka Daniel Iroegbu, Wangping Chen, Xun Wu, Ming Wu, Jinfu Yang}

Department of Cardiovascular Surgery, Second Xiangya Hospital, Central South University, Changsha 410011, China

Contributions: (I) Conception and design: CD Iroegbu; (II) Administrative support: J Yang; (III) Provision of study materials or patients: J Yang; (IV) Collection and assembly of data: All authors; (V) Data analysis and interpretation: CD Iroegbu; (VI) Manuscript writing: All authors; (VII) Final approval of manuscript: All authors.

Correspondence to: Jinfu Yang. Department of Cardiovascular Surgery, Second Xiangya Hospital, Central South University, Middle Renmin Road 139, Changsha 410011, China. Email: yjf19682005@csu.edu.cn.

Background: Endomyocardial fibrosis (EMF) is a neglected cardiovascular disease of poverty which carries a poor prognosis with no specific treatment affecting mainly children and young adults. Here, we report our 10 -year experience in the therapeutic management and surgical treatment for EMF.

Methods: From February 2009 to 2019 March, 55 patients diagnosed with EMF from our cardiology unit underwent surgical repair at our department's pediatric surgical division. There were 35 male, and 20 female patients whose ages varied from 1 year 2 months to 12 years mean age $5.7( \pm 3.2)$. We designed the study aimed at assessing the cardio-structural abnormalities and coronary vascular changes faced with EMF patients using echocardiography, and coronary angiography with a detailed and thorough surgical examination of each case.

Results: Of the 55 operated patients, 1 had mild lesions, 26 had moderate lesions, and 28 had severe heart disease. All but one patient was in NYHA functional class III or IV at the time of surgery. All but one female patient with mild ventricular lesions and no valvular involvement had severe atrioventricular valve regurgitation with valves considered suitable for both replacements; 45 patients mean age $6.0( \pm 3.1)$ and repair nine patients mean age $3.8( \pm 2.9)$. The mean endocardial thickness was $3,000( \pm 1519) \mu \mathrm{m}$.

Conclusions: The echocardiographic changes corresponded well to the findings on surgery and histopathology. The coronary changes seen included a spectrum of fibrin deposition, medial sclerosis and degeneration, and the formation of plexiform lesions. Surgically evaluating the resected cardiac tissue might help improve disease management.

Keywords: Endomyocardial fibrosis (EMF); endemic disease; endocardial stripping; genetic susceptibility; heart failure (HF); health services accountability

Submitted Dec 09, 2019. Accepted for publication Feb 07, 2020.

doi: $10.21037 / \mathrm{cdt} .2020 .02 .10$

View this article at: http://dx.doi.org/10.21037/cdt.2020.02.10

\section{Introduction}

Endomyocardial fibrosis (EMF) initially described during episodes of rapidly progressive heart failure (HF) usually associated with atrioventricular-valve regurgitation or from autopsies of patients who died from an advanced disease (1-3) is cardiomyopathy, that predominantly affects children and young adults. Although its etiology, pathogenesis, and natural history remains unclear, the disease is the most common cause of restrictive cardiomyopathy, characterized by an impaired diastolic filling of either one or both ventricles as a result of the fibrous tissue deposition on the endocardial surface, variable degree of systolic dysfunction, atrioventricular valve dysfunction, and associated intracavitary thrombi. Its physiology leads to the right and left HF, or even both carrying a significantly poor prognosis, with an estimated survival of 2 years after diagnosis. With no currently specific drug therapy, surgery (valvular repair/ replacement) though technically challenging and not available in most endemic areas corrects hemodynamic 


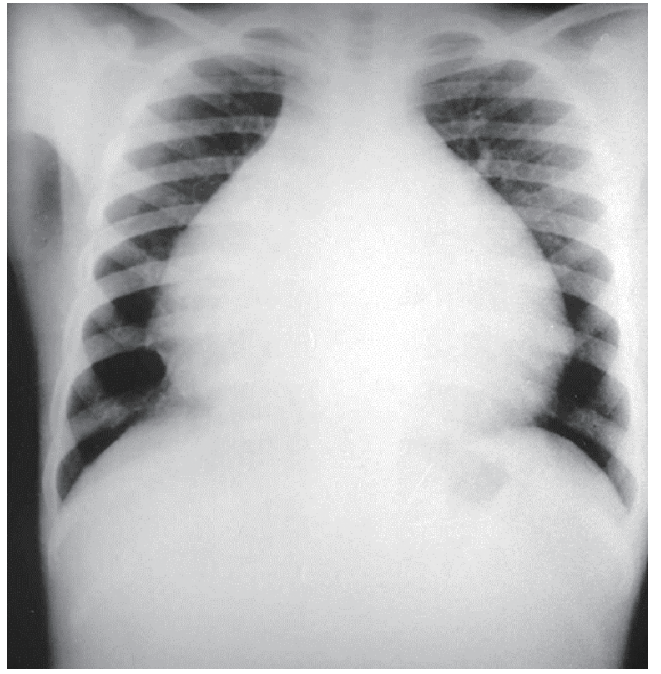

Figure 1 Preoperative chest X-ray features of advanced endomyocardial fibrosis of one of the older children (11 years old boy) showing marked cardiomegaly.

abnormalities thereby, improving the quality of life $(4,5)$ for all patients in NYHA III or IV and the resection of the thickened endocardium.

The use of cardiac magnetic resonance imaging (MRI) helps provides the information such as; presence or the absence of thrombi's, and the degree of myocardial involvement given a chance for non-invasive monitoring of the disease's progression and prognostication (6). However, its availability in endemic areas is meager, where the diagnosis is based on echocardiography. Irrespective of the part of the world one lives, EMF remains a challenging and mysterious cardiovascular disease poorly understood since its first description in 1948 with its variable phenotypic expression (depending on the stage at which it's been diagnosed) leading to HF and sudden death in children and adolescents (7). In this study, histopathological and surgical findings were compared to those obtained by echocardiography.

We designed the study aimed at assessing the cardiostructural abnormalities and coronary vascular changes faced with EMF patients using both transthoracic echocardiography (TTE) (preoperatively), and transesophageal echocardiography (TEE) (intraoperatively), with a detailed and thorough surgical examination of each case. We further evaluated the histopathological, and histomorphological changes in tissue(s) obtained from the excisional biopsies after surgery using both routine and immunohistochemical methods, with clinical correlation.

\section{Methods}

\section{Study population}

A scoring system was derived and based on the echocardiographic assessment of structural lesions and hemodynamic manifestations (echocardiography scoring system-ESS). The derived ESS was used to determine the severity of the fifty-five patients from our cardiology unit who all underwent surgical repair at our department's pediatric surgical division. Although, structural lesions and hemodynamic manifestations do not solely influence the disease state, gender, growth, age, and body composition are all confounding factors considered for this population. The scoring system used was based on the standardize quantification methods of the Pediatric Measurements Writing Group, The American Society of Echocardiography and the European Association of Echocardiography (7-10). The echocardiographic severity is scored as follows: grade I = 4-6 points, $\mathrm{II}=7-9$ points, and grade III $=10-12$ points. Patients considered for surgery include those presenting with recurrent symptoms, and grades II and III (moderate and severe) forms of the disease despite optimal medical therapy. Fifty-four patients in NYHA functional class III or IV were deemed suitable for operation, while one female patient who was in NYHA functional class II was observed for seven months before surgery (left ventricular endomyocardial decortication). Preoperative care included: (I) medical management of atrial arrhythmias and HF (II) treatment of concomitant infections and diseases; (III) drainage of pericardial, pleural and peritoneal effusions; and (IV) thrombotic risk and monitoring of eosinophilia. All patients were in a poor general condition, with an arguably low nutritional state. All fifty-five patients presented with suspicion for hypereosinophilia (number of eosinophils $\geq 450 \mathrm{LL}^{-1}$ ) and were placed on a short course of oral corticosteroids, angiotensin-converting enzyme (ACE) inhibitors and diuretics.

Auscultatory findings in all patients were unremarkable. Chest X-ray films showed enlargement of the heart in all cases (Figure 1). Electrocardiogram disclosed specific abnormalities. Signs of HF (which was defined as the presence of signs or symptoms associated with objective evidence of cardiac dysfunction. It could either be a left ventricular ejection fraction of $<50 \%$ and a raised aminoterminal pro-brain natriuretic peptide $>125 \mathrm{pg} / \mathrm{mL}$ ) with 
atrial fibrillation constituting the majority in forty-three patients with right bundle branch block found in seven patients, and a first-degree atrioventricular block is seen in three patients. Two patients showed evidence of left ventricular hypertrophy. Due to the atypical chest pain complaints made by the much more older children (above five years of age), cineangiography study was carried out to detect possible vascular lesions. During cineangiography, the vessel number per unit of area of the endocardium (square millimeter) was counted under medium power magnification $(\times 200)$ using a computerized image analysis system (Quantimet, Leica). The data were compared using the $t$-test, and differences were considered to be statistically significant when $\mathrm{P} \leq 0.05$. The initial group classification of patients for valvular repair or replacement was determined echocardiographically (TTE) in the catheterization laboratory. The echocardiographic analysis was based on cardiac chamber size and function, structural evaluation, and the assessment of additional hemodynamic parameters such as pulmonary artery pressure. The final group classification was carried out intraoperatively using TEE which heavily relied on the color Doppler jet characteristics, jet area, flow convergence, and the extent and distribution of fibrosis. Valve repair was opted for less fibrotic valve, while replacement for dysplastic and entirely fused valves which were both based on the surgical team discretion. The selection criteria used for bioprostheses and mechanical valve was based on the difficulties faced with anticoagulant therapy, annular size, limited valve selection, somatic growth, unavoidable reoperation, and high postoperative mortality $(11,12)$. Surgical contra-indications included ventricular arrhythmias, and a marked reduction in ventricular size, massive ascites, pulmonary hypertension, and extremely extensive parietal calcification.

\section{Surgical technique}

All patients underwent surgery through a median sternotomy, using conventional cardiopulmonary bypass lasting from 60 to 160 minutes, with a mean of $115.4( \pm 32.4)$. The intracardiac procedure was performed during a period of ischemic arrest. Myocardial protection was achieved using cold potassium cardioplegic solution, infused into the aortic root, and topical hypothermia of the heart. The duration of ischemic arrest ranged from 40 to 100 minutes, with a mean of $72.2( \pm 19.3)$. Endocardial decortication was accomplished in sixteen left ventricles alone, twenty-five right ventricles alone, and fourteen from both right and left ventricular chambers of the heart due to their biventricular involvement. A large right atriotomy was performed in all cases. Only fourteen cases were not carried out via routine atriotomy alone due to their biventricular involvement throughout the entire ventricular cardiac chambers. The remnants of the tricuspid valve were resected, and the fibrous tissue incised below the tricuspid annulus. The dissections were directed towards the ventricular apex into the posterior wall, allowing the resection of an entire fibrous shell. In all cases where right atriotomy was not employed, a good exposure was obtained via a large vertical left atriotomy. The left ventricular endocardial fibrosis was resected using the same guidelines as those employed for the right side and a mitral valvar prosthesis inserted. After decortication had been completed, valvar repair or replacement with a prosthetic was used. In this retrospective study of 55 pediatric patients, one patient had a left ventricular decortication without valvular repair nor replacement; 45 patients received valve replacement $(36$ patients had a bioprostheses while nine patients received a mechanical valve) while nine patients underwent valvular repair.

\section{Intra-operative evaluation}

The distribution, presence, and the extent of the atrioventricular valvar abnormalities were evaluated intraoperatively (Figure 2).

\section{Tissue sampling and handling}

The incised endomyocardial biopsies were obtained from both ventricles. Fourteen cases were not performed via routine atriotomy only due to the severe fibrotic tissues distributed throughout the entire ventricular cardiac chambers. Only samples containing myocardial tissue were considered for inclusion for histology and immunostaining in the study. Tissues from fibrotic areas of the papillary muscles, and the posterior aspect of the left ventricular wall were taken. Both atrial tissues were also obtained from patients submitted to atrial reduction. Tissues from the right ventricle were collected from the trabecular and admission chamber.

\section{Sample processing and staining}

Specimens were routinely processed in light microscopy after being embedded in paraffin wax, fixed in $10 \%$ buffered 

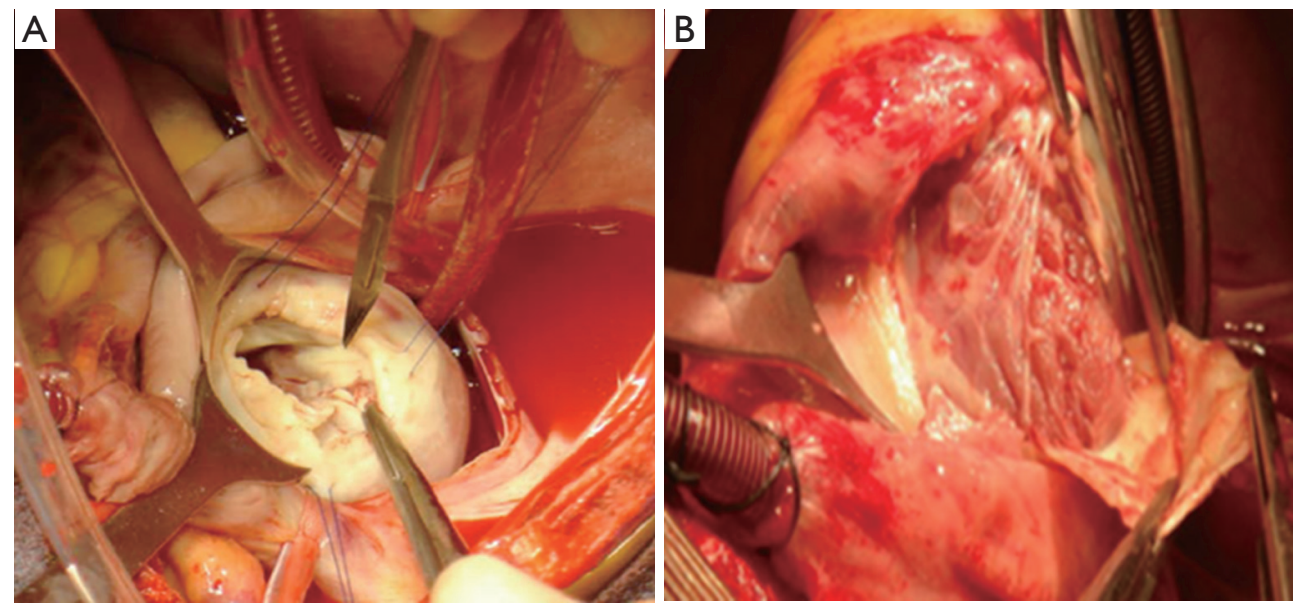

Figure 2 Intra-operative findings. (A) Surgical view of an endocardial resection from the ventricular cavity; (B) resection of the thickened endocardium reveals healthy looking myocardium underneath the fibrous tissue.

formalin, and cut into $4 \mu \mathrm{m}$ thick sections. The cut sections were stained for cell and tissue morphology (Hematoxylin $\&$ Eosin), mucins (Alcian Blue-Sirius Red), collagen content (Masson's Trichrome), and elastic fibers (Verhoeff ElasticVan Gieson). Eosinophils were identified using the carbol chromotrope technique.

\section{Definitions and histological analysis}

The targeted incisional endomyocardial biopsies were assessed for the location and presence of fibrosis, neovascularization, presence of inflammation, endothelial integrity, microvascular abnormalities, and evidence of myofibroblastic proliferation. Definitions used for microscopic evaluation of both atrial and ventricular tissues are the following.

\section{Inflammatory changes}

The intensity of the inflammation was classified as observed at high power magnification (HPM) as (I) rare ( $<5$ inflammatory cells per field of HPM); (II) mild (5-10 inflammatory cells per field of HPM); and (III) intense ( $>10$ inflammatory cells per field of HPM). Depending on the cell type present, the inflammatory changes were categorized as acute, chronic, or mixed while the inflammatory distribution was classified as either focal or diffused.

\section{Endocardial fibrosis}

The fibroblastic response type was classified as: (I) hyaline (moderate cellular response with massive plaque-like collagen); or (II) cellular (when cells were predominant in the background of loose fibrous tissue), or (III) mixed (the combination of both response type). Endocardial thickening was considered as mild, moderate, and severe when the thickness was $1,000,1,000-3,000$ or $3,000 \mu \mathrm{m}$, respectively using the JULIA software. The endocardial fibrosis was classified as diffuse or focal according to their distribution.

\section{Myocardial fibrosis}

The distribution and presence of excessive myocardial collagen was assessed and considered as being: replacement fibrosis (RF), fibrous tissues replacing group of myofibers; wide septa (WS), myofibers being separated by the extended subendocardial fibrosis; interstitial (IF), when the intercellular spaces are being affected; and perifascicular $(\mathrm{PF})$, the isolated group of myofibers.

\section{Immunostaining}

Paraffin-embedded formalin-fixed tissue sections were stained for the identification of endothelial cells and vessels (CD31, CD34), macrophages (CD68), leucocytes (CD45), and myofibroblasts (Smooth Muscle Actin, SMA) using the avidin-biotin-complex peroxidase technique (Figure 3).

\section{Patbological and clinical echocardiographic concordance}

We compared our immunohistological findings with the presence of severe blood eosinophilia, inflammatory 

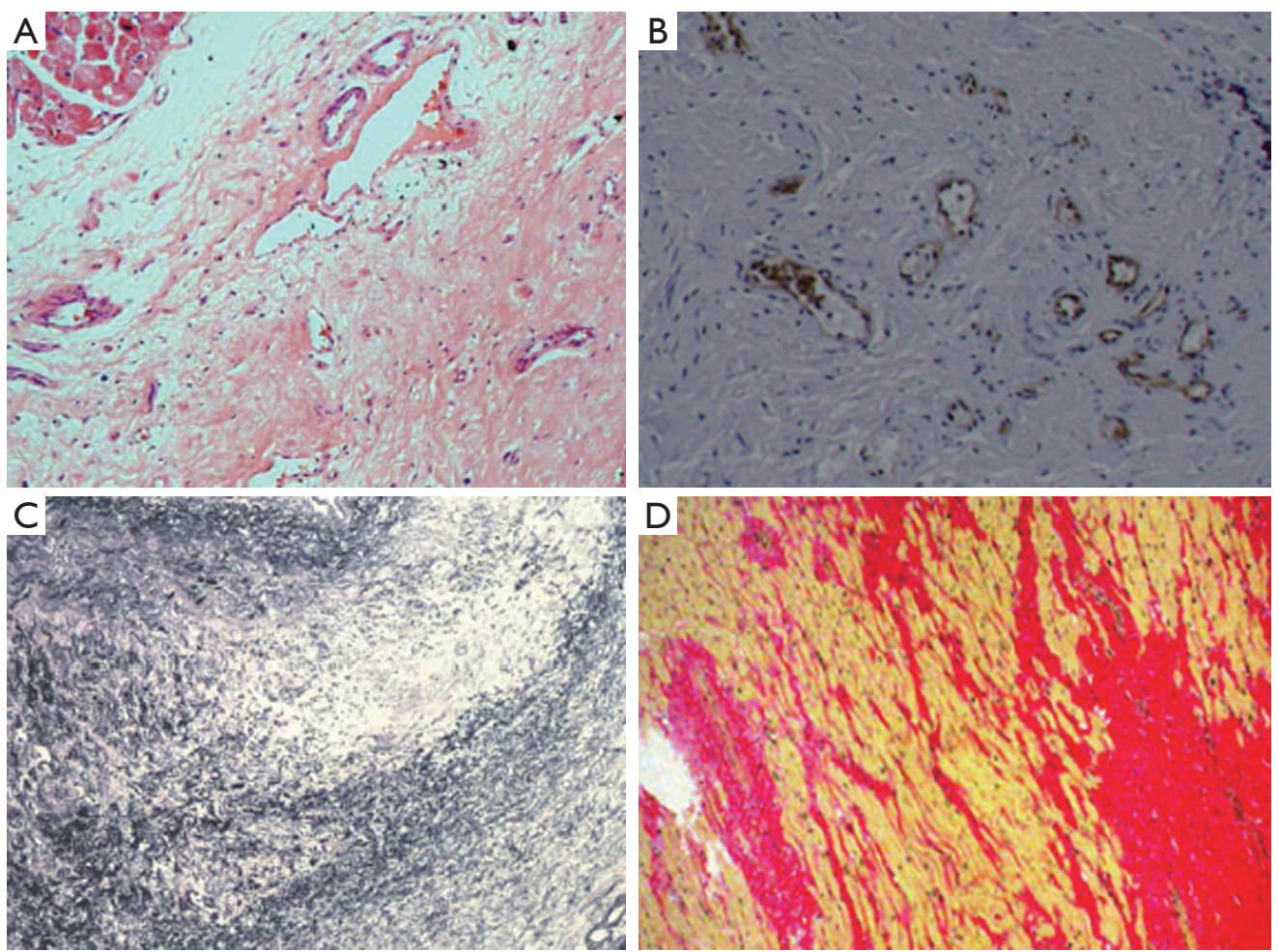

Figure 3 Histopathology and immunohistochemistry. (A) Microphotograph showing severe endocardial thickening, with a rich vascular net as well as groups of myocardial fibers separated from each other by strands of fibrous tissue extending from the subendocardium (H\&E, $\times 10)$; (B) hypervascularization in the sub-endocardium confirming the preservation of the lining of endothelial cells (CD31 positive cells) in the vessel wall [patchy cytoplasmic staining (dark bluish-brown) strong and more prone to background $\times 20$ ]; (C) proliferated elastic fibers at the superficial layer of the thickened endocardium (Verhoeff elastic stain $\times 40$ ); (D) magnification of strands of fibrous tissue penetrating the inner portion of myocardium and surrounding the blood vessels (Van Gieson $\times 20$ ).

laboratory markers, and the surgical results. Similarly, the concordance between surgical and echocardiographic results was assessed. Finally, a correlative clinical status was made by comparing the disease severity which was assessed by echocardiography to the pattern and intensity of myocardial and endocardial fibrosis.

\section{Statistical analysis}

Continuous variables and patient's demographic data are presented as mean \pm standard deviation.

\section{Ethical issues}

The ethical committee for Xiangya Second Hospital of Central South University approved the study. The patients' gave written informed consent for their wards to be included in the study.

\section{Results}

\section{Characterization of patients}

All but one female patient was in NYHA functional class III-IV at the time of surgery. The mean erythrocyte sedimentation rate was $(20.3 \mathrm{~mm} / \mathrm{h})$. The mean body mass index was $\left(19.3 \mathrm{~kg} / \mathrm{m}^{2}\right)$, and the mean absolute eosinophil count was $1.16( \pm 0.3)$ cells $/ \mathrm{mcL}$. Thirty-six patients had characteristic tissue hyper-eosinophilia (number of eosinophils $\geq 450 \mu \mathrm{L}^{-1}$ ). The relevant clinical and laboratory findings are presented in (Table 1).

\section{Echocardiography}

Of the 55 patients studied, one had mild lesions; 26 had moderate lesions ( 15 male, 11 female) while 28 had severe heart disease (20 male, 8 female). According to the distribution of cardiac lesions, EMF was predominantly 
Table 1 Relevant tissue collection sites from the 55 patients submitted to surgery with their relevant clinical and laboratory findings

\begin{tabular}{lc}
\hline Patient's relevant characteristics & $\mathrm{N}=55$ \\
\hline Mean age (years) & $5.7( \pm 3.2)$ \\
Sex (male/female) & $35 / 20$ \\
Mean body mass index $\left(\mathrm{kg} / \mathrm{m}^{2}\right)$ & $19.3( \pm 8.1)$ \\
Mean erythrosedimentation rate $(\mathrm{mm} / \mathrm{h})$ & $20.3( \pm 3.6)$ \\
Mean eosinophil count $($ cells/mcL) & $1.16( \pm 0.3)$ \\
NYHA class & \\
II & 1 \\
III/IV & $26 / 28$ \\
Classification & \\
Distribution: right/left/bilateral & $25 / 16 / 14$ \\
Severity: mild/moderate/severe & $1 / 16 / 36$ \\
Tissue obtained & \\
Right ventricle & \\
Left ventricle & None \\
Right atrium & 16 \\
Left atrium & None \\
\hline
\end{tabular}

right in twenty-five patients, left in sixteen patients, and biventricular in fourteen patients. Although, few discrepancies existed between TTE and TEE (all based on the premise). TTE could not be able to give a definite diagnosis for mitral valve regurgitation in some patients compared to TEE. This however, could be a result of the lateral decubitus positioning of the patient in the laboratory unless otherwise indicated. TEE on the other hand, was able to give a definite and precise diagnosis of mitral valve regurgitation in all cases. This apparent discrepancy could be related to the positioning of the patient (supine), the use of high-frequency imaging transducer which has a superior spatial resolution providing better images, and its multiplanar ability (multiplanar view) to cut and create the arbitrary oriented planes.

\section{Surgical data}

At the start of operation (sternotomy), moderate pericardial effusion (high in protein content and increased cellularity with a predominance of lymphocytes which tested negative for tuberculosis) could be seen as well as cardiomegaly due to atrial dilatation with varying degree of notching of the right border in patients with right-sided EMF. Further, the atria were invariably dilated while the ventricles showed endocardial and valvular lesion abnormalities in varying proportions. Patchy whitening and thickening of the endocardium were present in all patients. Atriotomy revealed a thickened atrial wall due to an edematous hypertrophied muscular layer. Intracardiac thrombosis was seen in twelve right atriums, fifteen left atriums, eleven right ventricles, six left ventricles, while eleven patients had no intracardiac thrombi. In twenty-five patients with right-sided EMF, the tricuspid annulus was markedly dilated giving ample access to the ventricular cavity, while the other sixteen patients with left-sided EMF all had moderate to the severe dilated mitral annulus. The mitral valve leaflets showed all diffuse irregular thickening. Left ventricular lesions were assessed by gently retracting the valve apparatus in all cases. The posterior leaflet, posterior papillary muscle, and the chordae tendinea's were partially or entirely fused to the posterior wall in most of the cases. The significant (moderate-severe) mitral regurgitation seen with TEE intraoperatively (before surgery) was as a result of the restricted leaflet mobility caused by the fusion of the anterior papillary muscle (Figure 4).

\section{Surgical findings}

All but one female patient with mild lesions from this retrospective study had severe atrioventricular valve regurgitation. Of the forty-five patients who all had valve replacement, valvular dysfunction was the main reason leading to an indication for surgery. Thirty-six patients received a bio-prosthesis (the so-called predominantly valvular forms) while the other nine who all received a mechanical valve replacement had mostly restrictive forms, with large thickened endocardial plaques, smallsized ventricles, and a significantly decreased compliance, as assessed by Doppler. The remaining nine patients with valvular repairs all had combined lesions with severe atrioventricular valve regurgitation and large fibrotic plaques.

\section{Echocardiographic features, histopathology and immunobistochemistry}

Six patients had no tissue inflammation. None of our patients had or developed bacterial endocarditis from the 

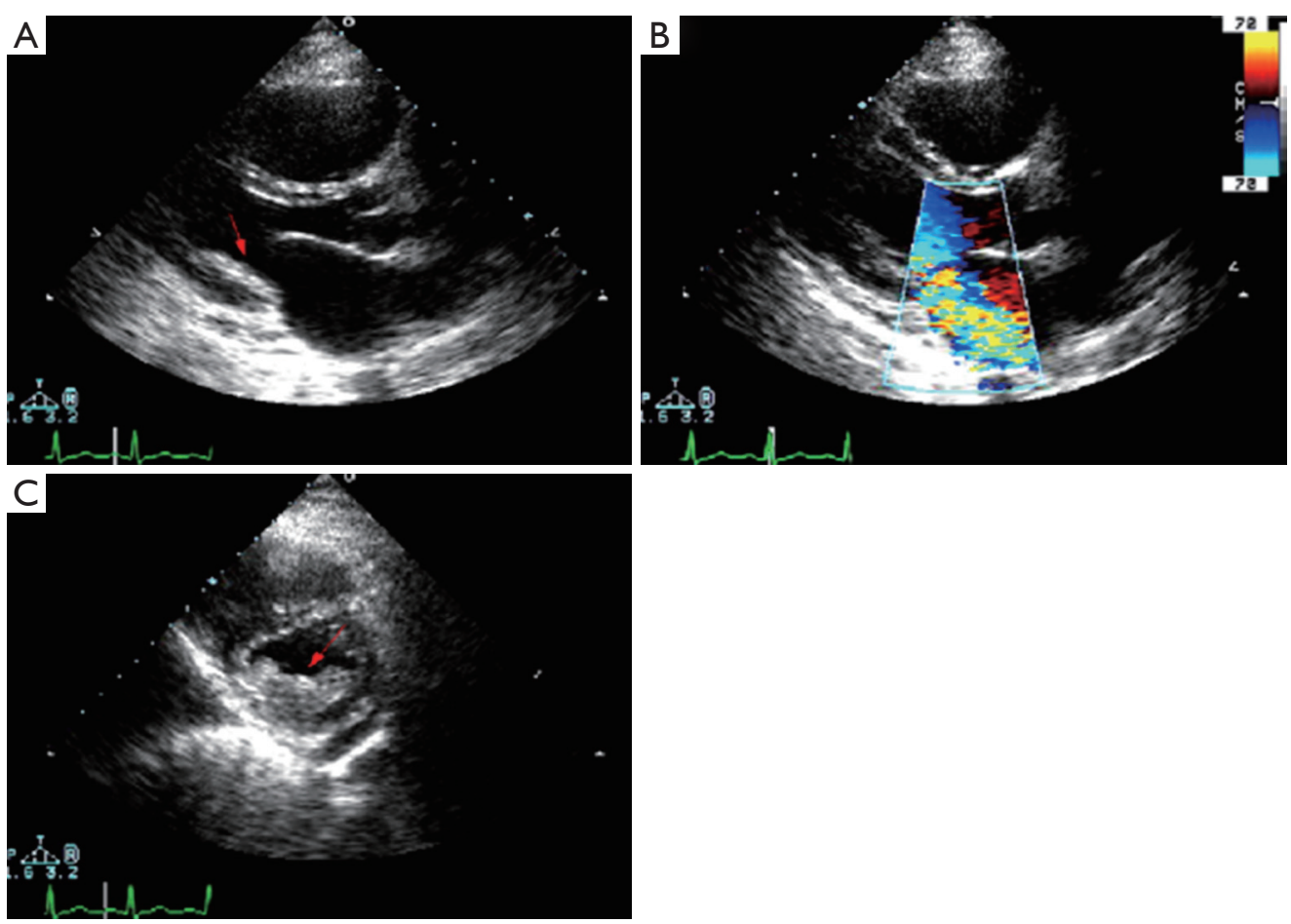

Figure 4 Echocardiogram of one of the older children (11 years old boy) with an advance endomyocardial fibrosis. (A) In the parasternal long-axis view, an area of increased echo density (arrow) denotes thrombus or fibrosis along the basal posterior wall of the left ventricle that also involves the posterior leaflet of the mitral valve; (B) color Doppler flow mapping in the same view demonstrates significant mitral regurgitation; $(\mathrm{C})$ in the short-axis view shown during diastole, there is extensive thrombus or fibrosis on the posterior wall (arrow) with compromise of the left ventricular cavity size.

time of admission to the day of discharge. As accessed by echocardiography, no patient had mild endocardial thickening. Severe endocardial thickening $(>3,000 \mu \mathrm{m})$ was present in 11/21 and 15/34 patients classified as moderate and severe EMF by ESS. Endocardial thickening was the most prominent abnormality, related to the deposition of cellular and hyaline collagen underneath a layer of apparently healthy endocardial endothelial cells. We evaluated tissue(s) from twenty-five right ventricles, sixteen left ventricles, and the other fourteen tissues evaluated were from patients having a biventricular EMF. The main findings in the excised tissue are presented in (Table 2). Endocardial fibrosis was due to an increased number of fibroblasts in the ventricular subendocardium (Figure 4A). Maximum endocardial thickness was 5,591 $\mu \mathrm{m}$, with a mean of $3,000 \pm 1,519 \mu \mathrm{m}$. Deep in the sub-endocardium, there were mixed scanty inflammatory infiltrates, intense foci of neovascularization, and capillary channels. Deep in the sub-endocardium mainly composed of lymphocytes, mononuclear cells, macrophages, and plasma cells
(Figure 4B). The proliferation of elastic fibers at the superficial layer with the inner myocardium was penetrated by strands of fibrous tissue (Figure $4 C$ ). Degenerative myocardial changes included enlarged bizarre nuclei, myocyte hypertrophy, and myocardial disarray often concerning scars.

\section{Vascular findings}

Vascular lesions were found mainly within the areas of EMF, appearing as broad septa penetrating the myocardium from the densely scarred endocardium. Only coronary arteries and arterioles were involved. For the purposes of description, the findings would be considered under the following heading.

\section{Medial degeneration}

Medial swelling was frequently observed particularly at the sites of vascular bifurcation. There was swelling of the muscle cell cytoplasm, with or without vacuolation. 
Table 2 Histological abnormalities of ventricular tissue samples surgically obtained from 55 patients with EMF

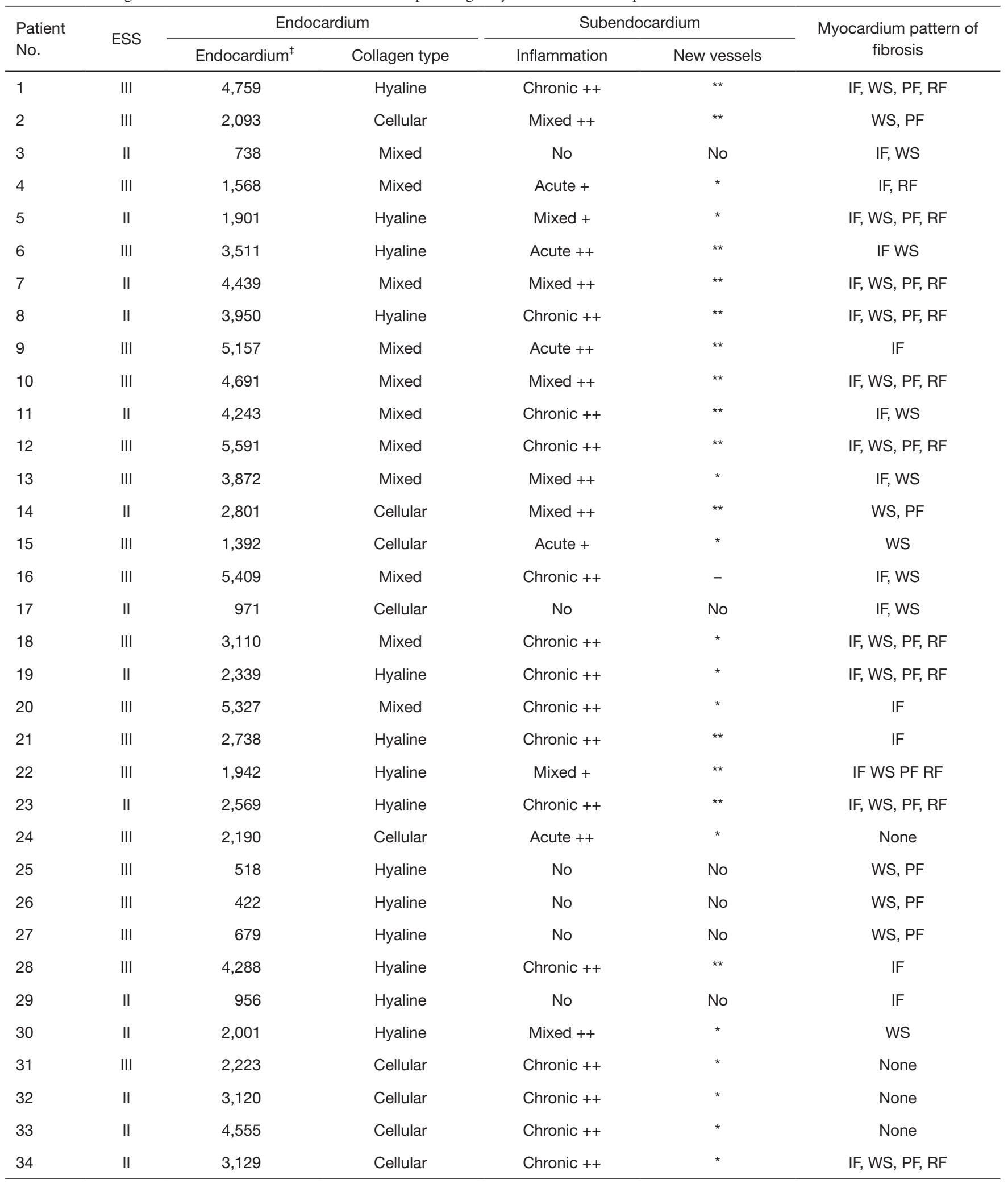

Table 2 (continued) 
Table 2 (continued)

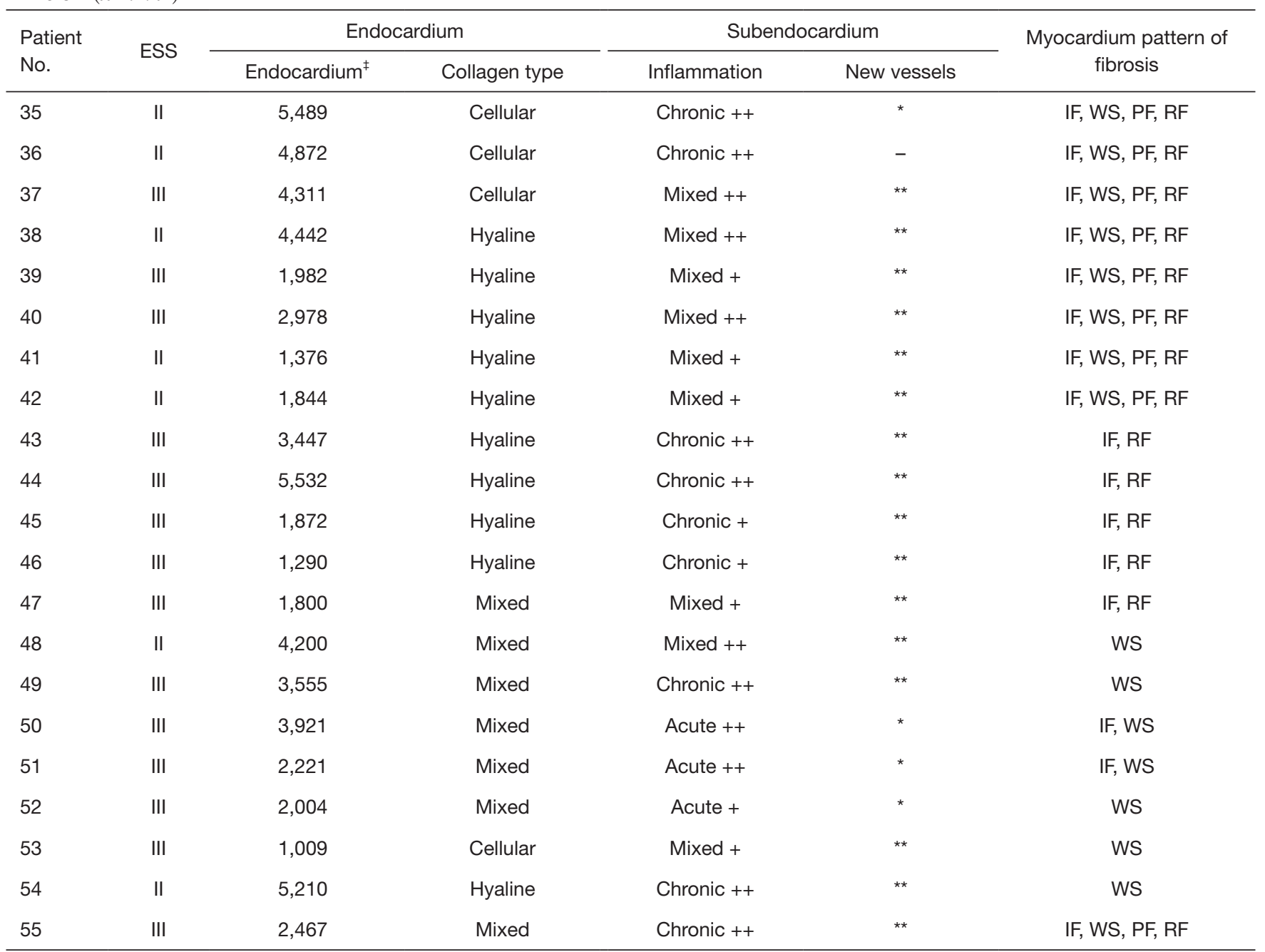

Number of eosinophilic cells in the ventricular subendocardium (acute $>500-700$, chronic $>870-1,000$, mixed $>1,000$ numerous inflammatory infiltrates predominantly eosinophilic), Presence of more than one infiltrative cell-type such as lymphocytes, macrophages, plasma cells, and fibroblastic cells in the ventricular subendocardium $(+>1,++>2)$. Degree of highly foci patterned neovascularization $\left(^{*}\right.$, poorly neovascularized; **, moderately neovascularized). No (no new vessels), - (indefinite), none (no specific identified myocardial pattern of fibrosis), ${ }^{\ddagger}$, institutional values. ESS, echocardiography severity score; PF, perifascicular fibrosis; WS, wide septa; IF, interstitial fibrosis; RF, replacement scars.

\section{Medial focal proliferation}

Focal areas of medial degeneration or fibrosis were observed seen in some medium-sized arteries.

\section{Medial sclerosis}

Replacement of the medial muscle cells by fibrous tissue was seen in several arteries and arterioles in every case.

\section{Intimal proliferation}

This appeared to be associated with spurs projecting into the lumen with fibrinous materials (intimal bridges) mainly on the endothelial surface of arteries of various calibers.

\section{Other changes}

Other changes found where congestion, perivascular edema, and a slight perivascular lymphocytic accumulation.

\section{Selective cineangiography}

Due to the procedural complications (arrhythmia, pseudoaneurysm, and especially bleeding) encountered 
Table 3 Concordance between echocardiographic and surgical findings

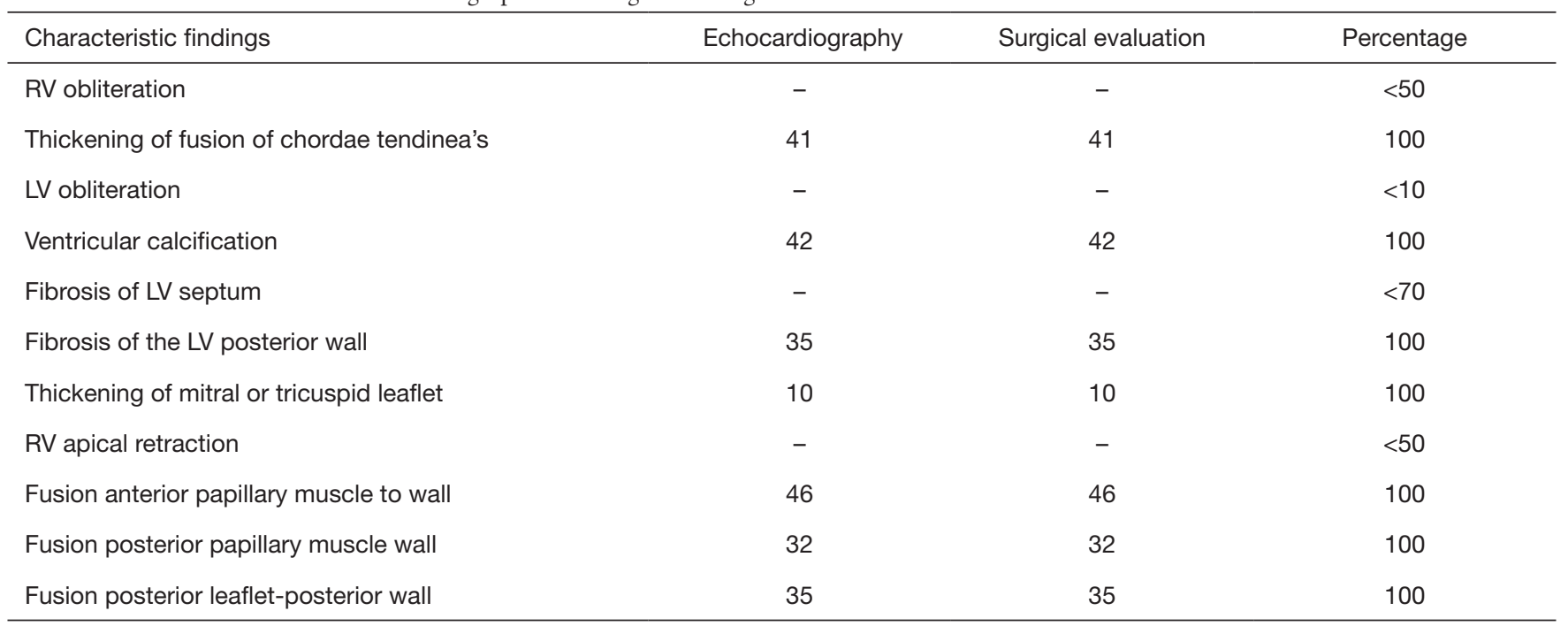

earlier (arguably due to technical experience) in twentyfour patients between the age of one year eight months and four years two months with mean age 2.9, catheterization was only performed for patients with the age five years and above. Diagnostic selective cineangiography of the right-sided cavities showed atrial dilation, tricuspid regurgitation, and an abnormal filling of the apex of the affected ventricle. Left ventricular cineangiography revealed mitral regurgitation and obliteration of the apical wall.

\section{Concordance between echocardiographic and surgical data}

The concordance echocardiographic and surgical findings for each patient were absolute for fibrosis of the LV posterior wall, fusion anterior papillary muscle to the wall, a fusion of the posterior papillary muscle and leaflet to the wall, ventricular calcification, thickening of mitral or tricuspid leaflets, and thickening or fusion of chordae tendinea (Table 3). We were, however, unable to echocardiographically and surgically find the coinciding relationship wherein: (I) fibrosis of the $\mathrm{LV}$ posterior wall; (II) LV apical fibrosis; (III) RV apical retraction; and (IV) LV and RV obliteration.

\section{Concomitant conditions}

No concomitant defect(s) were discovered intraoperatively. Also, none of the patients had or showed any signs of active rheumatic carditis or infective endocarditis.

\section{Outcomes}

There was no death during the postoperative period. Fortytwo patients showed "early" postoperative complications of HF such as low cardiac output, atrial fibrillations, and a complete atrioventricular block, while twelve patients showed no signs of "early" postoperative complications immediately after surgery, ("early" one to three days after surgery). Twenty-six patients did not clinically improve six days after the operation, eight patients required reoperations for valvular dysfunctions and vastly poor coronary perfusion, while the much older twenty patients (7-12 years of age) clinically improved wherein NYHA class I (8) and II (12) postoperatively. An approach through the left atrium and the mitral valve was chosen to minimize the potential for additional trauma caused by the exposure itself for the re-operated patients. During reoperation, the incompetent valves were resized and replaced; and internal decortication of the ventricular wall was performed (peeling off the fibrotic and thickened endocardium) to restore compliance of the underlying myocardial tissue, improve coronary perfusion, and cardiac output (ejection fraction). Low cardiac output, poor coronary perfusion largely due to fibrotic compression of the coronary vessels, valve incompetence (prostheses), and arrhythmias (ventricular) where the findings preventing clinical improvements in the re-operated patients.

Eleven patients, which were part of the much older patient's group (seven male patients and four female patients) were each followed up for a period of three years. 
Of the eleven patients followed-up, echocardiographic evidence showed improvement of hemodynamics and ventricular function in the four female patients. Out of the seven male patients followed-up, four patients developed recurrent mitral regurgitation, while three showed echocardiographic signs of EMF "possible" reoccurrence on contralateral side with poor perfusion, and hemodynamics (low cardiac output), and atrial fibrillation. More than half of the patients in our study were from the up-state rural countryside and neighboring provinces. Due to the relatively poor economic conditions and financial status of most of our patients, we were unable to carry out post-operative follow-up studies of forty-three patients. Of the forty-three patients who were not followed-up, four patients died of sudden cardiac arrest (mostly toddlers; 1-year 2 months to 1 -year 11 months) while thirteen showed severe signs of HF (both verbally communicated).

\section{Family bistory}

Most of the patients when asked about their family history of premature death, $\mathrm{n}=23$ patient guardians complained of an "unusual" death of a loved one, distant and also close relatives after a brief illness between the ages of three and fifteen. In their own words They stated that, before their death, the relative complained of high body temperature (fever), troubled sleep (insomnia), difficulty breathing (dyspnea) sometimes on exertion, chest-pounding (abnormal heartbeat), weight loss, general body weakness (fatigability), occasional loss of consciousness, swollen face and feet (facial and pedal edema), and an abdominal swelling (ascites). The other patient guardians $(n=32)$ were not sure about the premature death cases.

\section{Discussion}

An endemic neglected cardiovascular disease of poverty, when encountered in children and toddlers, is a serious disease. Although, surgical treatment is far less satisfactory than one would wish as observed in the study; EMF can be accurately diagnosed by echocardiography (more precisely TEE). This study shows a high concordance between echocardiography and the pathological surgical findings. The findings in the study also further show that, evaluation of surgically resected pathological cardiac tissue and immunohistochemistry support the diagnosis of EMF. EMF is common among children and young adults; although, its incidence in children is difficult to establish $(13,14)$.
Children are at a disadvantage compared to adults in a way that they normally have a greater life expectancy $(15,16)$. The clinical presentation of the toddlers and children in our study with EMF seems not to differ from that of adults $(17,18)$. The endocardial ventricular thickening detected on echocardiography corresponded to the thick whitish endocardial walls and the valve apparatus surgically observed in variable extensions. The atypical chest pain complaints which were mostly described by the much older children in our study has been mentioned by some authors and linked to the pathological alterations in the left ventricle (19-21). Although, we were unable to characterize the chest pain complaints made by the children; the clinical impression is that, it might be a result of an altered cardiac microcirculation.

During surgery, mild to moderate inflammatory infiltrates were seen to be scattered predominantly in the interface between the endocardium and the myocardium as previously described in the literature (1-3). We were able to identify and characterize tissue eosinophilia in thirty-six patients in our study who all had hyper-eosinophilia at the time of admission favoring the eosinophilic etiopathogenic factor of the disease (22-24). This has however, led some authors to associate EMF with the Löeffler syndrome (Löeffler endocarditis), where endocardial fibrosis and hyper-eosinophilia (idiopathic hyper-eosinophilic syndrome-HES) can be present $(25,26)$. Although, HES is a sporadic disorder, it is commonly diagnosed in much older patients between the ages of 20 and 50 years old $(27,28)$. It should also be known that; EMF is a feature of multiple diseases and Non-compaction of the ventricular myocardium should also be considered as a possible predisposing factor. It is most frequent in the general population and the only known entity with a similar distribution to EMF and/or its associated cardiomyopathies (mostly restrictive type) with a poor sub-endocardial coronary flow $(29,30)$.

As reported in a study by Jaiyesimi, the patchy fibrotic reoccurrence and atrial stretching encountered during the reoperation of eight patients in our study, was due to the progressive atrial dilatation, valvular dysfunctions, and poor coronary perfusion (31). This however, serves as a basis for the origin and sustainability of atrial arrhythmias in EMF. This postoperative setback, however, highlights the high morbidity and mortality rate associated with the disease alongside the possible reoccurrence of EMF lesions on the contralateral side of the affected cardiac chamber $(32,33)$. From the results of the immunohistological and pathological 
resected patients' cardiac tissue in our study (hyaline and acellular collagen), EMF seems to be an interstitial disease. The preserved endothelial endocardium, and the multifocal chronic inflammatory cascade affect different areas of the same heart suggesting the present ongoing injury or temporally spaced insults $(34,35)$. In addition, the lack of vessels in the outer endocardium in our study may explain why bacterial endocarditis did not occur in any patient. This however, collaborates the pathological criterion of differentiating rheumatic heart diseases from $\operatorname{EMF}(36,37)$. As already described and reported in the literature $(38,39)$, diagnostic coronary cineangiocardiography in our study, demonstrated the typical ventricular deformities of the disease.

Furthermore, the presence of coronary vascular lesions in EMF is not unexpected; as can be seen in our study; the vascular lesions were frequent and varied. Due to its frequency, we considered medial degeneration to be the primary vascular lesion, among others. Medial degeneration in coronary arteries, with subsequent sclerosis and calcification, has been recognized in man and experimental animals under several different conditions $(40,41)$. In our study, however, the vascular lesions did not seem to be secondary to the EMF itself. It also did not appear to represent a primary feature of the disease, since there was no correlation found between them and the degree of endomyocardial scarring. It seems to us that the probable vascular changes observed may be caused by the same obscure factor(s) that leads to EMF. Regardless of their unclear etiology, we considered those obstructive coronary arterial lesions that were a prominent histopathological feature in our study as a pathognomonic element in the progression of this disease.

The downhill course of patients with EMF is its poor postoperative outcome and grave prognosis which is no different in children $(42,43)$, as seen in our study. The early age of clinical diagnosis (congenital), and low nutritional state observed in all patients in our study has been reported in literature to contribute to the pathogenesis of EMF (44-47). It's also mentioned that malnutrition may contribute to the pathogenesis of EMF by increasing susceptibility to parasitic infections $(48,49)$. This would however, cause a dysfunction in eosinophils regulatory mechanisms, which would eventually lead to necrosis, and finally fibrosis $(50,51)$. Although, there are still, however, no affirmative evidence to firmly establish these hypotheses. Medical therapy such as corticosteroids, ACE inhibitors and diuretics administered in our patients are usually ineffective, and the only chance for survival is surgical treatment $(52,53)$. However, the long-term outcome from medical treatment in advanced stages is very poor with $75 \%$ mortality at 2 years $(42,54-56)$. Hence, open heart surgery (OHS) should be performed before irreversible cardiac and hepatic damage occurs $(57,58)$. Although, access to OHS is severely limited in many endemic areas, as seen in our study; it variably increases the chance of survival. Surgery consists of mitral valve replacement or repair and conservative endocardectomy (endocardial stripping) (59-62). Another viable alternative to endocardectomy with $\mathrm{OHS}$ is a heart transplant which improves the surgical outcomes especially in patients with clinical signs of an advanced EMF $(63,64)$.

Finally, as seen with our study, EMF seems to be an evolving disease; as some patients continue to present with EMF even in postoperative periods either as a recurrence in the operated ventricle or the previously normal one (65). Also, some patients do not improve clinically after the operation as observed in more than half of our patients in this study. Another fact that may adversely affect the postoperative course, especially in children, is the dysfunctioning of the valvar prostheses. Hence, every effort should be made to preserve the native valve during the operation. Unfortunately, despite recognizing this imperative, it did not prove to be possible as we inserted prostheses in all patients.

\section{Study limitation}

We acknowledge the fact that our study had some limitations: during histopathological analysis of the specimens, the focal hemorrhage observed could actually be from the operative procedure. Also, the differentiation between WS and PF might have been compromised by the tangential cuts made on the tissue. Finally, the autopsy of the four patients who died of sudden cardiac death (personal communication) was not ascertained to determine the cause of death (EMF reoccurrence or related poor postoperative outcome).

\section{Conclusions}

An endemic neglected disease of poverty, EMF still remains one of the most poorly understood and mysterious cardiovascular disorders today. Structural abnormalities of EMF can accurately be diagnosed by echocardiography precisely by TEE. The evaluation of surgically resected cardiac tissues from the affected patients with the 
histopathological and histochemical analysis of the tissue specimen helps back the echocardiographic diagnosis. The laboratory findings further improve the pathophysiological understanding of the disease and the obstructive coronary arterial lesions which were a prominent histopathological feature in our study. Finally, the role of surgery in improving the knowledge and understanding of the disease must not and should not be overlooked despite the poor long-term outcomes.

Efforts should now be concentrated on increasing its medical awareness among health workers, and elucidating the etiology of the disease, which might permit the establishment of preventive measures.

\section{Acknowledgments}

Funding: None.

\section{Footnote}

Conflicts of Interest: All authors have completed the ICMJE uniform disclosure form (available at http://dx.doi. org/10.21037/cdt.2020.02.10). The authors have no conflicts of interest to declare.

Ethical Statement: The authors are accountable for all aspects of the work in ensuring that questions related to the accuracy or integrity of any part of the work are appropriately investigated and resolved. The ethical committee for Xiangya Second Hospital of Central South University approved the study. The patients' gave written informed consent for their wards to be included in the study.

Open Access Statement: This is an Open Access article distributed in accordance with the Creative Commons Attribution-NonCommercial-NoDerivs 4.0 International License (CC BY-NC-ND 4.0), which permits the noncommercial replication and distribution of the article with the strict proviso that no changes or edits are made and the original work is properly cited (including links to both the formal publication through the relevant DOI and the license). See: https://creativecommons.org/licenses/by-nc-nd/4.0/.

\section{References}

1. Connor DH, Somers K, Hutt MS, et al. Endomyocardial fibrosis in Uganda (Davies' disease) Part I. Am Heart J
1967;74:687-709.

2. Connor DH, Somers K, Hutt MS, et al. Endomyocardial fibrosis in Uganda (Davies' disease) Part II. Am Heart J 1968;75:107-24.

3. Harris LC, Nghiem QX. Cardiomyopathies in infants and children. Prog Cardiovasc Dis 1972;15:255-87.

4. Iglezias SD, Benvenuti LA, Calabrese F, et al. Endomyocardial fibrosis: pathological and molecular findings of surgically resected ventricular endomyocardium. Virchows Arch 2008;453:233-41.

5. de Oliveira SA, Pereira Barreto AC, Mady C, et al. Surgical treatment of endomyocardial fibrosis: a new approach. J Am Coll Cardiol 1990;16:1246-51.

6. Cheung SC, Chan CWS. Insights of prognostication of Davies disease: What could we learn from serial magnetic resonance imaging studies? Int J Cardiol 2010;142:e32-4.

7. Celermajer DS, Chow CK, Marijon E, et al. Cardiovascular disease in the developing world: prevalences, patterns, and the potential of early disease detection. J Am Coll Cardiol 2012;60:1207-16.

8. Buck T, Plicht B, Erbel R. Current recommendations on echocardiographic evaluation of the severity of mitral regurgitation: standardization and practical application using a scoring system. Herz 2006;31:30-7.

9. Lopez L, Colan SD, Frommelt PC, et al. Recommendations for Quantification Methods During the Performance of a Pediatric Echocardiogram: A Report From the Pediatric Measurements Writing Group of the American Society of Echocardiography Pediatric and Congenital Heart Disease Council. J Am Soc Echocardiogr 2010;23:465-95.

10. Nunes MC, Tan TC, Elmariah S, et al. Impact of Incorporating Commissural Morphology and Leaflet Displacement to the Prediction of Outcome for Patients Undergoing Percutaneous Mitral Valvuloplasty. Circulation 2014;129:886-95.

11. El Oakley R, Kleine P, Bach DS. Choice of Prosthetic Heart Valve in Today's Practice. Circulation 2008;117:253-6.

12. Cetinkaya A, Poggenpohl J, Bramlage K, et al. Longterm outcome after mitral valve replacement using biological versus mechanical valves. J Cardiothorac Surg 2019;14:120.

13. Sezi CL. Current status of endomyocardial fibrosis in Uganda. In: Valiathan MS, Sommers K, Kartha CC, editors. Endomyocardial fibrosis. Delhi: Oxford University Press, 1993:11-5.

14. Ikeme AC, et al. Natural history and current status in Nigeria. In: Valiathan MS, Sommers K, Kartha CC. 
editors. Endomyocardial fibrosis. Delhi: Oxford University Press, 1993:29-36.

15. Ferreira MB. Endomyocardial fibrosis in Mozambique. Paris: Paris V University-Rene Descartes, 2000

16. Sliwa K, Damasceno A, Mayosi BM. Epidemiology and etiology of cardiomyopathy in Africa. Circulation 2005;112:3577-83.

17. Rutakingirwa $M$, et al. Poverty and eosinophilia are risk factors for endomyocardial fibrosis in Uganda. Trop Med Int Health 1999;4:229-35.

18. Kartha CC. Endomyocardial fibrosis: a case for the tropical doctor. Cardiovasc Res 1995;30:636-43.

19. Gupta PN, Valiathan M, Balakrishnan KG, et al. Clinical course of endomyocardial fibrosis. Br Heart J 1989;62:450-4.

20. Barretto AC, Mady C, Pileggi F. Longitudinal followup of patients not treated by surgery. Arq Bras Cardiol 1996;67:285-8.

21. Bestetti RB, Corbucci H, Fornitano L, et al. Anginalike chest pain and syncope as the clinical presentation of left ventricular endomyocardial fibrosis: a case report. Angiology 2005;56:339-42.

22. Andy JJ, Ogunowo PO, Akpan NA, et al. Helminth associated hypereosinophilia and tropical endomyocardial fibrosis (EMF) in Nigeria. Acta Trop 1998;69:127-40.

23. Frustaci A, Abdulla AK, Possati G, et al. Persisting hypereosinophilia and myocardial activity in the fibrotic stage of endomyocardial disease. Chest 1989;96:674-5.

24. Patel AK, D'Arbela PG, Somers K. Endomyocardial Fibrosis and eosinophilia. Br Heart J 1977;39:238-41.

25. Brockington IF, Olsen EGJ. Loeffler's endocarditis and Davies' endomyocardial fibrosis. Am Heart J 1973;85:308-22.

26. Noguchi H, Kephart GM, Colby TV, et al. Tissue eosinophilia and eosinophil degranulation in syndromes associated with fibrosis. Am J Pathol 1992;140:521-8.

27. Jin X, Ma C, Liu S, et al. Cardiac involvements in hypereosinophilia-associated syndrome: Case reports and a little review of the literature. Echocardiography 2017;34:1242-6.

28. Allderdice C, Marcu C, Kabirdas D. Intracardiac Thrombus in Leukemia: Role of Cardiac Magnetic Resonance Imaging in Eosinophilic Myocarditis. CASE (Phila) 2018;2:114-7.

29. Nucifora G, Aquaro GD, Pingitore A, et al. Myocardial fibrosis in isolated left ventricular non-compaction and its relation to disease severity. Eur J Heart Fail 2011;13:170-6.

30. Kalavakunta JK, Tokala H, Gosavi A, et al. Left ventricular noncompaction and myocardial fibrosis: a case report. Int Arch Med 2010;3:20

31. Jaiyesimi F. Controversies and advances in endomyocardial fibrosis: a review. Afr J Med Med Sci 1982;11:37-46.

32. Somers K. Restrictive Cardiomyopathies. In: Pongpanich B, Sueblinvong V, Vongprateep C. editors. Pediatric Cardiology. International Congress Series 906. Excerpta Medica., Amsterdam, 1990.

33. Mocumbi AO. Endomyocardial fibrosis: A form of endemic restrictive cardiomyopathy. Glob Cardiol Sci Pract 2012;2012:11.

34. Radhakumary C, Kumari TV, Kartha CC. Endomyocardial fibrosis is associated with selective deposition of type I collagen. Indian Heart J 2001;53:486-9.

35. Adams DH, Lloyd AR. Chemokines: leucocyte recruitment and activation cytokines. Lancet 1997;349:490-5.

36. Shaper AG, Hutt MSR, Coles RM. Necropsy study of endomyocardial fibrosis and rheumatic heart disease in Uganda 1950-1965. Br Heart J 1968;30:391-401.

37. Somers, K, D'Arbela, PG, Patel, AK. Endomyocardial fibrosis. In: Shaper, AG, Kibukamusoke, JW, Hutt, MSR, eds. Medicine In a Tropical Environment. London: British Medical Association, 1972:348.

38. Balakrishnan KG, Sasidharan K, Venkitachalam CG, et al. Coronary angiographic features in endomyocardial fibrosis. Cardiology 1983;70:121-6.

39. Fernandes F, Mady C, Vianna Cde B, et al. Radiological findings in endomyocardial fibrosis. Arq Bras Cardiol 1997;68:269-72.

40. James TN. Pathology of small coronary arteries. Am J Cardiol 1967;20:679.

41. Rahlf G. Microscopic Pathology of Intramural Coronary Arteries and Arterioles of the Left Ventricle in Arterial Hypertension. In: Strauer BE. editor. The Heart in Hypertension. New York: Springer-Verlag, 1981.

42. D'Arbela PG, Mutazindwa T, Patel AK, et al. Survival after first presentation with endomyocardial fibrosis. Br Heart J 1972;34:403-7.

43. Parry EH, Abrahams DG. The natural history of endomyocardial fibrosis. Q J Med 1965;34:383-408.

44. Davies H. Endomyocardial fibrosis and the tuberous diet. Int J Cardiol 1990;29:3-8.

45. Kumari KT, Ravikumar A, Kurup PA. Accumulation of glycosaminoglycans associated with hypomagnesaemia in endomyocardial fibrosis in Kerala: possible involvement of dietary factors. Indian Heart J 1997;49:49-51.

46. Kuruvilla L, Kartha CC. Cerium depresses endocardial endothelial cell-mediated proliferation of cardiac 
fibroblasts. Biol Trace Elem Res 2006;114:85-92.

47. Connor DH, Somers K, Nelson AM, et al. The cause of endomyocardial fibrosis in Uganda. Trop Doct 2012;42:206-7.

48. Mocumbi AO, Falase AO. Recent advances in the epidemiology, diagnosis and treatment of endomyocardial fibrosis in Africa. Heart 2013;99:1481-7.

49. Aiello VD, Jatene MB. Early presentation of endomyocardial fibrosis. Cardiol Young 2011;21:474.

50. Freers J, Mayanja-Kizza H, Rutakingirwa $M$, et al. Endomyocardial fibrosis: why is there striking ascites with little or no peripheral oedema? Lancet 1996;347:197.

51. Sivasankaran S. Restrictive cardiomyopathy in India: the story of a vanishing mystery. Heart 2009;95:9-14.

52. Mocumbi AO, Yacoub S, Yacoub MH. Neglected tropical cardiomyopathies, II: endomyocardial fibrosis: myocardial disease. Heart 2008;94:384-90.

53. Falase AO. Endomyocardial fibrosis in Africa. Postgrad Med J 1983;59:170-178.

54. Russo PA, Wright JE, Ho SY, et al. Endocardectomy for the surgical treatment of endocardial fibrosis of the left ventricle. Thorax 1985;40:621-5.

55. Tharakan J, Bohora S. Current perspective on endomyocardial fibrosis. Curr Sci 2009;97:405.

56. Bukhman G, Ziegler J, Parry E. Endomyocardial fibrosis: still a mystery after 60 years. PLoS Negl Trop Dis 2008;2:e97.

57. Mocumbi AO, Sidi D, Vouhe P, et al. An innovative

Cite this article as: Iroegbu $\mathrm{CD}, \mathrm{Chen} \mathrm{W}, \mathrm{Wu} \mathrm{X}, \mathrm{Wu} \mathrm{M}$, Yang J. Endomyocardial fibrosis. Cardiovasc Diagn Ther 2020;10(2):208-222. doi: 10.21037/cdt.2020.02.10 technique for the relief of right ventricular trabecular cavity obliteration in endomyocardial fibrosis. J Thorac Cardiovasc Surg 2007;134:1070-2.

58. Cherian SM, Jagannath BR, Nayar S, et al. Successful reoperation after 17 years in a case of endomyocardial fibrosis. Ann Thorac Surg 2006;82:1115-7.

59. Nair U, Evans T, Oakley D. Surgical treatment of endomyocardial fibrosis with preservation of mitral valve. Br Heart J 1980;43:357-9.

60. Moraes F. Surgery for endomyocardial fibrosis revisited. Eur J Cardiothorac Surg 1999;15:309-12.

61. Uva MS, Jebara VA, Acar C, et al. Mitral valve repair in patients with endomyocardial fibrosis. Ann Thorac Surg 1992;54:89-92.

62. Tang A, Karski J, Butany J, et al. Severe mitral regurgitation in acute eosinophilic endomyocarditis: repair or replacement? Interact Cardiovasc Thorac Surg 2004;3:406-8.

63. Korczyk D, Taylor G, McAlistair H, et al. Heart transplantation in a patient with endomyocardial fibrosis due to hypereosinophilic syndrome. Transplantation 2007;83:514-6.

64. de Freitas HF, de Castro PP, Chizzola PR, et al. Heart transplantation in a patient with endomyocardial fibrosis. Arq Bras Cardiol 2005;84:49-50.

65. Moraes F, Lapa C, Hazin S, et al. Surgery for endomyocardial fibrosis revisited. Eur J Cardiothorac Surg 1999;15:309-12; discussion 312-3. 